\title{
Intervenção interdisciplinar enquanto estratégia para o U so Racional de Medicamentos em idosos
}

\author{
An interdisciplinary intervention \\ as a strategy for Rational Use of Drugs by the elderly
}

Eloá Fátima Ferreira M edeiros ${ }^{1}$

Clayton Franco M oraes ${ }^{1}$

M auro Karnikowski ${ }^{2}$

Otávio Toledo Nóbrega ${ }^{3}$

M argô Gomes de Oliveira Karnikowski

${ }^{1}$ Programa de PósGraduação stricto sensu em Gerontologia, Universidade Católica de Brasília. QS, 07. 71966-700 Brasília DF. eloa_medeiro@yahoo.com.br ${ }^{2}$ Secretaria deSaúdedo Distrito Federal.

3 Programa de Pós-

Graduação em Ciências

M édicas, U niversidade de Brasília.
Abstract This research assessed the effectiveness of interdisciplinary interventions involving physicians, pharmacists and nutritionists, aimed at the promotion of the Rational $U$ se of Drugs. It is a study of a prospective and analytical nature conducted with a population of elderly females, where the effectiveness of the intervention was assessed according to indicators of the Rational Use of Drugs established by the World Health Organization. Statistical analysis was performed using the $t$ test or the one way AN OVA for discrete variables, as well as the chi-square test for categorical analyses. After the intervention, an average reduction in drug consumption was observed in comparison with the research performed prior to the intervention $(p=0.001)$. The drugs used in cardiovascular therapy were those consumed most, which tallies with the diseases mentioned by the elderly. It was seen that interdisciplinary intervention among the elderly can contribute to improve rational drug use indicators, especially prescription drugs. Key words Elderly, Drugs, Indicators, Interdisciplinary healthcare team
Resumo A presente pesquisa avaliou a efetividade de intervenções interdisciplinares, envolvendo médicos, farmacêuticos e nutricionistas, destinadas à promoção do U so Racional dos M edicamentos. Trata-se de um estudo de caráter prospectivo e analítico, com uma população demulheres idosas, onde a efetividade da intervenção foi avaliada de acordo com indicadores de U so Racional dos M edicamentos preconizados pela Organização M undial de Saúde. As análises estatísticas foram realizadas utilizando-se do testet ou one-way AN OVA para variáveis discretas, eo teste de qui-quadrado para avaliação categórica das variáveis. Após a intervenção, houve uma redução média no consumo demedicamentos em relação às consultas da préintervenção ( $p=0,001)$. Os medicamentosutilizadosna terapêutica cardiovascular foram osmais consumidos, o que se encontra em consonância com as doençasautoreferidas pelasidosas. Foi possível verificar que a intervenção interdisciplinar em idosas pôde contribuir para melhoria dosindicadores de U so Racional dos M edicamentos, em especial os de prescrição.

Palavras-chave Idoso, Medicamento, Indicadores, Equipe interdisciplinar de daúde 


\section{Introdução}

Os estudos demográficos evidenciam um crescente aumento da população idosa no Brasil eno mundo. É estimado que a população idosa cresça mundialmente mais de $80 \%$ nos próximos 25 anos ${ }^{1}$. Atualmente, no Brasil, os idosos representam mais de 18 milhões de pessoas, correspondendo a $10,5 \%$ da população, sendo que os idosos com mais de 80 anos alcançaram $1,4 \%$ do contingente brasileiro ${ }^{2}$. Desta forma em menos de 40 anos, o Brasil passou de um perfil de mortalidade típico de uma população jovem para um quadro caracterizado por enfermidades crônicas emúltiplas, próprias das faixas etárias mais avançadas ${ }^{3}$. Em geral, estas doenças exigem acompanhamento de uma equipe desaúde multidisciplinar, com intervenções contínuas além da necessidade de introdução de terapia farmacológica, gerando custos diretos e indiretos mais elevados e maior atenção de sua família e da sociedade.

0 padrão de consumo elevado de medicamentos entre os idosos que vivem na comunidade tem sido descrito tanto no Brasil e no mundo ${ }^{4-7}$. Em média, 2 a 5 medicamentos são prescritos regularmente a idosos ${ }^{8}$ e a prevalência de uso émaior entreas mulheres independentemente da faixa de idadet.

É consenso que o desenvolvimento de medicamentos representa um grande avanço na história da ciência e que contribui com relevante significância para a mel horia da qualidade devida da população ${ }^{9}$. No entanto, a possibilidade de um dano induzido em decorrência da utilização de fármacos, mesmo quando utilizados nas doses preconizadas e com indicação terapêutica adequada se constitui em fato real ${ }^{10}$.

A população idosa possui risco elevado de problemas relacionados a medicamentos devido às alterações fisiológicas naturais relacionadas ao envelhecimento associado à maior incidência de múltiplas doenças crônicas e ao grande número de medicamentos consumidos ${ }^{11-13}$.

A vulnerabilidade dos usuários de medicamentos, em especial os idosos, torna-se pronunciada quando se pratica o uso indiscriminado de medicamentos. A utilização dos medicamentos envolvendo mau uso e abuso de consumo, e a não adesão a tratamentos importantes tem provocado impacto sobre as medidas públicas para prevenção de agravos e promoção da saúde, assim como sobre o ciclo econômico envolvido na prestação dos serviços de saúde ${ }^{14}$. A Organização Mundial da Saúde (OMS) estima que mais da metade de todos os medicamentos são pres- critos, dispensadosou vendidos inadequadamente, e que, aproximadamente $50 \%$ de todos os pacientes não os utilizam corretamente ${ }^{15}$.

Ao longo do século XX, o medicamento deixou de ser somente um instrumento de intervenção terapêutica para converter-seem um elemento complexo - técnico e/ou simbólico - na socie dade ocidenta| ${ }^{16}$. Atualmente a prescrição farmacoterapêutica tornou-se quase que obrigatória nas consultas médicas, sendo o médico avaliado pelo paciente por esta prática. Assim, a prescrição do medicamento tornou-sesinônimo de boa conduta médica, justificando sua enorme demanda ${ }^{17}$.

Portanto, a medicalização da vida reforça a necessidade por uma abordagem multidimensional do atendimento, pautada no modelo interdisciplinar aplicado ao envel hecimento ecujo foco está no sujeito da intervenção. Frigotto ${ }^{18}$ definea interdisciplinaridade como uma necessidade relacionada à realidade concreta, histórica e cultural, constituindo-se assim em questão ético-política, econômica, cultural e epistemológica. No campo da saúde, a interdisciplinaridade acena como a possibilidade da compreensão integral do ser humano no contexto das relações sociais e do processo saúde-doença, rompendo com a fragmentação entre saberes e práticas ${ }^{19}$. Esta pesquisa se propôs avaliar a efeti vidade da intervenção interdisciplinar, direcionada ao idoso, objetivando a promoção do U so Racional dos M edicamentos.

\section{M ateriais e métodos}

Este estudo de caráter prospectivo elongitudinal foi realizado em uma população de mulheres de 60 anos ou mais, residente no Distrito Federal, Brasil, einscritas no Projeto Promoção da Saúde do Idoso da Universidade Católica de Brasília (UCB). Através de meios de divulgação e palestras-convites foram cadastradas 130 mulheres idosas, as quais foram conduzidas à consulta médica, nutricional efarmacêutica contituindo a amostra total de todas as etapas do estudo.

0 estudo foi dividido em duas fases: pré-intervenção e pós-intervenção. A pré-intervenção constituiu-se de duas consultas, sendo a primeira no ano de 2005 ea segunda em 2006/2007. 0 esquema de procedimentos adotado encontraserepresentado na Figura 1. Os objetivos da préintervenção foram definir aspectos sócio-econômicos das pacientes, investigar doen ças auto-referidas, bem como tomar conhecimento do perfil dos medicamentos utilizados pela população 


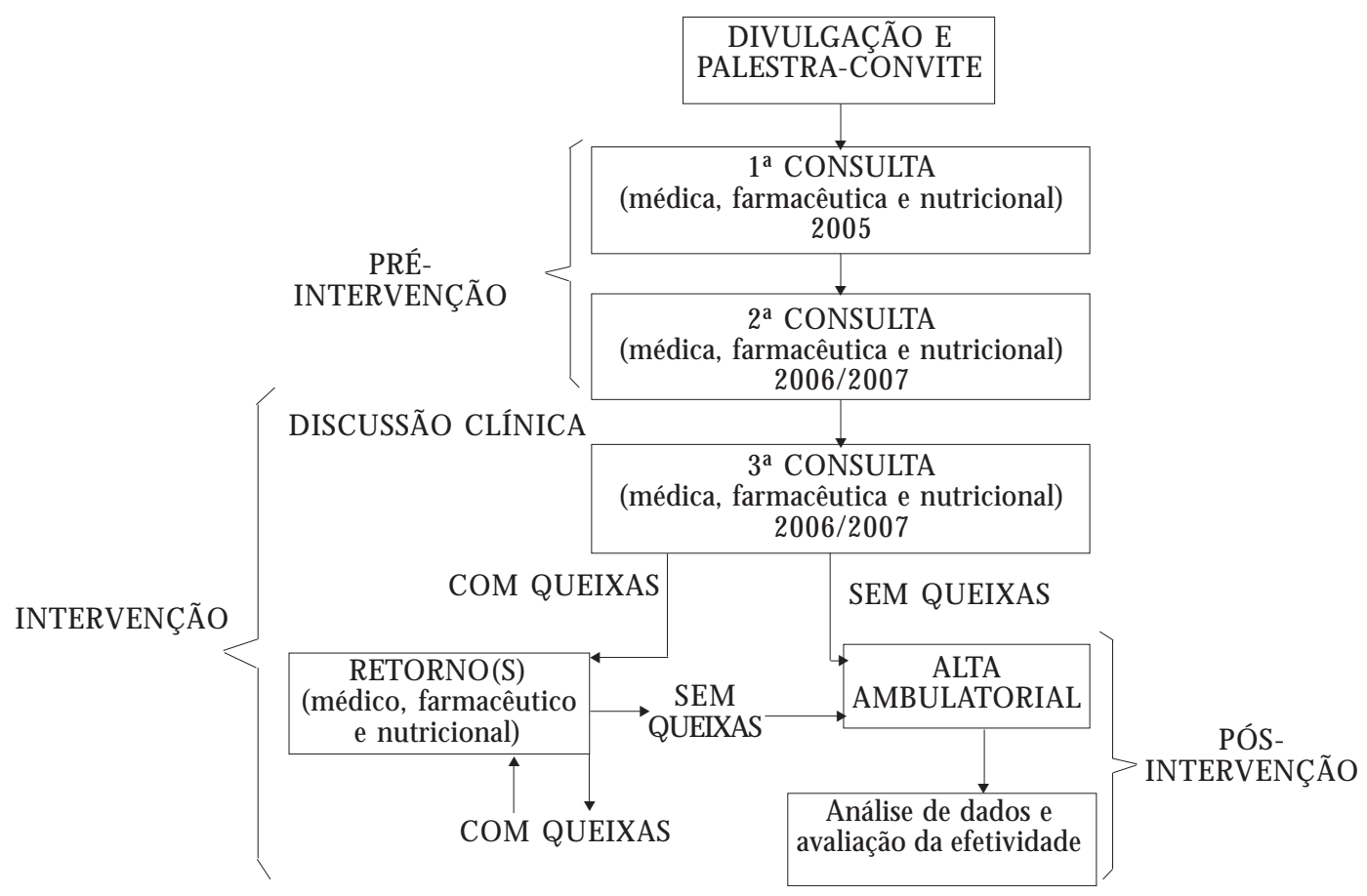

Figura 1. Fluxograma de atendimento interdisciplinar a uma população de idosas.

em estudo. A pós-intervenção ocorreu em 2006/ 2007, sendo os objetivos desta fase a realização das intervenções interdisciplinares e a avaliação da sua efeti vidade. 0 tempo entrea segunda consulta da pré-interven ção ea pós-interven ção não ultrapassou um mês.

Durante todas as consultas do estudo foi realizado um levantamento dos medicamentos utilizados e seu perfil de consumo.

\section{Aspectos sócio-econômicos}

Os aspectos sócio-econômicos investigados foram: faixa etária, renda familiar e o grau de escolaridade. A renda familiar foi estimada de acordo com salário mínimo $(R \$ 300,00)$ à época do estudo.

Levantamento

e perfil de consumo de medicamentos

Foram levantados todos os medicamentos referidos pelas pacientes, porém somente foram incluídos na análise os produtos farmacêuticos industrializados, homeopáticos efitoterápicos de uso crônico que possuíam composição de prin- cípios ativos claramente determinados. A Denominação Comum Brasileira ${ }^{20}$ foi utilizada para a identificação dos princípios ativos a partir dos nomes comerciais disponíveis. Os medicamentos foram classificados de acordo com o número de princípios ativos assim denominados: monodroga, bidroga e polidroga (quando possuíam acima de três princípios ativos). Os princípios ativos encontrados em cada medicamento foram agrupados em conformidade com o Sistema de Classificação Anatômico-TerapêuticoQuímico (ATC) ${ }^{21}$.

O consumo concomitante de dois ou mais princípios ativos quaisquer a um mesmo indivíduo, por um período ininterrupto de três meses ou mais foi considerado como polifarmácia ${ }^{22}$, sendo que polifarmácia maior foi definida como 0 consumo de 5 ou mais medicamentos ${ }^{23}$. Foi considerada automedicação o uso demedicamentos sem indicação de um profissional de saúde.

\section{Intervenção interdiciplinar}

As intervenções interdisciplinares foram determinadas caso a caso após discussão clínica, com a participação dos profissionais que com- 
punham a equipe interdisciplinar. A equipe interdisciplinar que atuou em todas as etapas da pesquisa foi composta por quatro médicos das especialidades de clínica médica, ginecologia, ge riatria e urologia, seis farmacêuticos das áreas de atuação de imunologia, farmacologia, atenção farmacêutica, farmácia comunitária, análises clínicas e farmácia hospitalar e quatro nutricionistas com formação em nutrição clínica. Dentreas intervenções realizadas priorizou-se a terapêutica não farmacológica e quando havia necessidade de terapia medicamentosa esta era embasada nas diretrizes terapêuticas nacionais e internacionais, de acordo com a prevalência das doenças auto-referidas e primando pelo Uso Racional de M edicamentos. Foram respeitadas as particularidades fisiopatológicas do idoso, e também na disponibilidade do medicamento na lista nacional demedicamentos essenciais (RENAM E), bem como a autonomia de prescrição do médico. $\mathrm{A}$ paciente recebeu alta ambulatorial quando cessou a queixa principal. Foram considerados 0 tempo de consulta médica e o retorno para avaliar o cuidado ao paciente durante intervenção.

\section{Efetividade da intervenção}

Para avaliar a efetividade da intervenção interdisciplinar foram utilizados os indicadores de URM preconizados pela Organização M undial de Saúde ${ }^{24}$ e registrados nos períodos pré e pósintervenção. Os indicadores de URM selecionados nesta pesquisa foram: prescrição, número de medicamentos por consulta, medicamentos prescritos por nome genérico ${ }^{25}$, medicamentos prescritos contidos nas listas de medicamentos essenciais da OM S ( 150 WHO M odel Formulary) ${ }^{26}$ e brasileira (REN AM E 2006) ${ }^{27}$. Foi incluído como indicador de URM a prescrição dos medicamentos impróprios a idosos, conforme descritos pelos critérios canadenses ${ }^{28,29}$.

Osindicadores de URM foram expressos utilizando-se a média ou porcentagem. Quando expressos em média, foram seguidos de desvio padrão tanto na pré quanto na pós-intervenção. Para determinar a relação entre o consumo total de medicamentos de uso crônico e os indicadores de prescrição foi utilizada a razão média. Para determinar a relação entre o consumo total de medicamentos de uso crônico e o consumo de medicamentos impróprios foi utilizada a diferença média.

\section{Análise estatística}

As análises estatísticas foram realizadas empregando-se o pacote de programas SPSS versão 10.0 for W indows, utilizando-se as seguintes técnicas: testet para amostras independentes, O neway AN OVA ou o teste não paramétrico de quiquadrado.

Esta pesquisa foi realizada após aprovação pelo Comitê de Ética e Pesquisa da U CB constante do ofício CEP /UCB 082/2004, de 25 de outubro de 2004.

\section{Resultados}

As mulheres idosas participantes deste estudo ( $n=130$ ) possuíam idademédia de $68,2 \pm 5,8$ anos com renda média de 2,1 salários mínimos, sendo que $60,8 \%$ ( $n=79$ ) da população estudada nunca freqüentou escola ou não concluiu ciclo fundamental deensino.

$\mathrm{Na}$ primeira consulta foi verificado que 114 das 130 idosas atendidas $(87,7 \%)$ consumiam 414 medicamentos de uso contínuo $(4,4 \pm 2,9$ medicamentos/idosa, amplitude 1 a 16), correspondendo a 492 princípios ativos sendo 115 sem repetição. A diferença entre o número de medicamentos e os princípios ativos consumidos ocorreu, pois $15 \%$ dos primeiros se constituem em associações de dose fixa de 2 ou mais princípios ativos: 41 bidrogas e 18 polidrogas. Na segunda consulta, 113 idosas (86,9\%) utilizavam 418 medicamentos de uso contínuo $(4,1 \pm 2,6$ medicamentos/idosa, amplitude 1 a 17) num total de 469 princípios ativos, sendo 137 sem repetição. Foram constatadas $10 \%$ de associações, das quais 22 eram bidrogas e 10 polidroga. Após a intervenção interdisciplinar realizada, 116 idosas $(89,2 \%)$ passaram a consumir 348 medicamentos de uso contínuo (3,1 $\pm 2,0$ medicamentos/ idosa, amplitude 1 a 14), num total de 370 de princípios ativos, edestes 90 sem repetição, sendo 16 bidrogas e 5 polidrogas.

Não houve diferença significativa em relação à média de medicamentos de uso contínuo consumidos nos dois momentos investigados anteriormente à interven ção. No entanto, após a intervenção houve uma redução média no consumo de medicamentos em relação às duas consultas da pré-intervenção $(p=0,001)$.

Na primeira e na segunda consulta da préintervenção, 101 e 98 idosas respectivamente, foram consideradas polimedicadas, edestas, $45,6 \%$ $\left(n_{\text {pré2005 }}=45\right)$ e $43,9 \%\left(n_{\text {pré2006/2007 }}=43\right)$ eram poli- 
medicadas maior. Após a intervenção, o número de idosas polimedicadas foi de 95 , sendo $24,7 \%$ $\left(n_{\text {pós2006/2007 }}=23\right)$ polimedicadas maior. A polimedicação mai or difere significativamente entre os momentos pré e pós-intervenção $\left(\div^{2}=11,74\right.$, $p=0,025)$, observando-se uma redução após a intervenção.

A classe de medicamentos mais consumida durante as fases prée pós-intervenção foi aquela relacionada ao sistema cardiovascular (Tabela 1), que se encontra em consonância com as doenças autoreferidas pelas idosas (Tabela 2). Cabe ressaltar que a hipertensão arterial, a dislipidemia e a insuficiência cardíaca, que são distúrbios de impacto sobre o sistema cardiovascular, totalizaram a maior parte das doenças autoreferidas $\left(\mathrm{n}_{\text {pré2005 }}=114 ; \mathrm{n}_{\text {pré2006/2007 }}=131\right.$ ).

Os princípios ativos mais utilizados foram a hidroclorotiazida $\left(n_{\text {pré2005 }}=42 ; n_{\text {pré2006/2007 }}=49\right.$; $\mathrm{n}_{\text {pós2006/2007 }}=49$ ), captopril ( $\mathrm{n}_{\text {pré2005 }}=21 ; \mathrm{n}_{\text {pré2006/ }}$ $\left.{ }_{2007}=26 ; n_{\text {pós2006/2007 }}=23\right)$, enalapril $\left(n_{\text {pré2005 }}=21\right.$; $\mathrm{n}_{\text {pré2006/2007 }}=18 ; \mathrm{n}_{\text {pós2006/2007 }}=28$ ), ácido acetil salicílico $\left(\mathrm{n}_{\text {pré2005 }}=17 ; \mathrm{n}_{\text {pré2006/2007 }}=28 ; \mathrm{n}_{\text {pós 2006/2007 }}=23\right) \mathrm{e}$ indapamida $\left(\mathrm{n}_{\text {pré2005 }}=15 ; \mathrm{n}_{\text {pré2006/2007 }}=17 ; \mathrm{n}_{\text {pós 2006/ }}\right.$ ${ }_{2007}=16$ ), todos utilizados nos distúrbios cardiovasculares. 0 alendronato $\left(n_{\text {pré2005 }}=11 ; n_{\text {pré2006/ }}\right.$ $\left.{ }_{2007}=12 ; n_{\text {pós 2006/2007 }}=12\right)$ e o cálcio $\left(n_{\text {pré2005 }}=13\right.$; $n_{\text {pré2006/2007 }}=15 ; n_{\text {pós 2006/2007 }}=18$ ), apesar de classificados pela ATC em grupos distintos, são princípios ativos utilizados em distúrbios ósseos, como a osteoporose, erelacionados entre os mais consumidos. Os grupos e subgrupos farmacológicos consumidos encontram-se descritos a seguir (Tabela 1).

Q uanto aos indicadores de prescrição observou-se que a relação entre medicamentos prescritos pelo nome genérico, os contidos nas listas nacional einternacional demedicamentos essenciais e o total de medicamentos prescritos no período pré e pós-intervenção apresentaram diferenças significativas (Tabela 3).

A diferença média entre 0 total de medicamentos prescritos e os medicamentos imprórios utilizados pela população idosa foi de $3,4( \pm 2,8)$ naprimeira consulta e $3,3( \pm 2,5)$ na segunda consulta, no momento pré-intervenção. Após a intervenção esta diferença média foi de $2,7( \pm 2,0)$, ressaltando-se que houve diferença significativa $(p<0,05)$ entre ambos os momentos pré e pós. Existiu uma correlação entre a polimedicação ea utilização demedicamento impróprio $\left(\chi^{2}=8,11 \mathrm{e}\right.$ $p=0,05$ ).

Na primeira consulta, anterior à intervenção, uma entre quatro pacientes consumia algum medicamento considerado impróprio à popula- ção idosa. N este momento foram 47 especialidades farmacêuticas em um conjunto de 20 princípios ativos impróprios, sendo que a fluoxetina, amiodarona, amitriptilina, metildopa, digoxina e diazepam, todos princípios ativos impróprios conforme os critérios de Beers (2003) encontravam-sena REN AME. Na segunda consulta $23,1 \%$ $(n=30)$ idosas consumiam 35 produtos farmacêuticosimpróprios, sendo 16 princípios ativose permanecendo os mesmos 6 contidos na RENAME. Após a intervenção $13,8 \% \quad(n=18)$ utilizavam 19 especialidades impróprias sen do que dos 9 princípios ativos utilizados, foram mantidos a amitriptilina, amiodarona, digoxina, fluoxetina e metildopa contidas na RENAME.

Em relação ao tempo médio de consulta mé dica, foram obtidos 32,8 minutos para o primeiro atendimento e 14,7 minutos para os atendimentos de retorno no período de intervenção.

A automedicação foi adotada por $20,2 \%$ $(n=23)$ pacientes contemplando 30 especialidades farmacêuticas, sendo um evento total de 44 princípios ativos, dados estes observados durante a primeira consulta. Dentre os medicamentos consumidos por automedicação figuravam aque les que deveriam ser dispensadosapenas sob prescrição médica, correspondendo a 46,7\% (n=14) das especialidades farmacêuticas. $\mathrm{Na}$ segunda consulta pré-interven ção, 10,6\% ( $n=12$ ) das idosas fizeram automedicação com 17 especialidades farmacêuticas, compondo um evento total de 21 princípios ativos, sendo que destes, $35,3 \%(n=6)$ especialidades farmacêuticas só deveriam ser dispensadas mediante prescrição médica.

Dentreaqueles que possuem restrição devenda sem prescrição médica, o diclofenaco foi o princípio ativo mais utilizado por automedicação nas duas consultas pré intervenção investigadas, correspondendo a $9,1 \%(n=4)$ e $11,8 \%$ $(n=2)$ respectivamente. Os medicamentos fitoterápicos e homeopáticos apresentaram frequência de consumo por automedicação de 26,6\% $(n=8)$ na primeira consulta e $52,9 \% \quad(n=9)$ na segunda consulta pré-intervenção investigadas.

\section{Discussão}

O perfil socio-econômico da população em estudo, mulheresidosas com baixa escolaridade erenda apresentando doenças crônicas não transmissíveis, reflete as condições sócio-demográficas eepidemiológicas dos países em desenvolvimento ${ }^{11,}, 30$.

Os resultados revelam, em consonância com os demais estudos s $^{31,32}$, que apesar da tentativa 
Tabela 1. Distribuição dos princípios ativos consumidos pela amostra de pacientes idosos conforme critérios do dicionário anátomo- químico-terapêutico (WHO, 2008).

\begin{tabular}{|c|c|c|c|c|c|c|}
\hline \multirow[b]{3}{*}{ Grupo anatômico terapêutico } & \multicolumn{4}{|c|}{ Pré-intervenção } & \multirow{2}{*}{\multicolumn{2}{|c|}{$\begin{array}{c}\text { Pós-intervenção } \\
\text { 2006/2007 } \\
\end{array}$}} \\
\hline & \multicolumn{2}{|r|}{2005} & \multicolumn{2}{|r|}{$2006 / 2007$} & & \\
\hline & $\%$ & $\begin{array}{l}\text { Principio ativo } \\
\text { por idosa }\end{array}$ & $\%$ & $\begin{array}{l}\text { Principio ativo } \\
\text { por idosa }\end{array}$ & $\%$ & $\begin{array}{l}\text { Principio ativo } \\
\text { por idosa }\end{array}$ \\
\hline $\begin{array}{l}\text { A - Trato alimentar e } \\
\text { metabolismo }\end{array}$ & 15,2 & 75 & 13,1 & 61 & 14,3 & 53 \\
\hline $\begin{array}{l}\text { Antiácidos, Antiflatulentos, } \\
\text { Anticolinérgicos, Antieméticos } \\
\text { e Antidiarréicos }\end{array}$ & 5,1 & 25 & 2,8 & 13 & 1,9 & 7 \\
\hline Fármacos hipoglicemiantes & 3,0 & 15 & 4,1 & 19 & 4,3 & 16 \\
\hline Vitaminas & 2,2 & 11 & 1,9 & 9 & 1,9 & 7 \\
\hline Suplementos minerais & 4,9 & 24 & 4,3 & 20 & 6,2 & 23 \\
\hline $\begin{array}{l}\text { B - Sangue e órgãos formadores } \\
\text { de sangue }\end{array}$ & 4,7 & 23 & 6,4 & 30 & 6,8 & 25 \\
\hline $\begin{array}{l}\text { Fármacos antitrombóticos e } \\
\text { preparações antianêmicas }\end{array}$ & 4,7 & 23 & 6,4 & 30 & 6,8 & 25 \\
\hline C - Sistema cardiovascular & 39 & 192 & 45,8 & 214 & 57,3 & 212 \\
\hline Cardioterápicos & 2 & 10 & 1,5 & 7 & 1,6 & 6 \\
\hline Antihipertensivos deação central & 1,6 & 8 & 1,5 & 7 & 2,2 & 8 \\
\hline Diuréticos & 14,2 & 70 & 16,9 & 79 & 19,7 & 73 \\
\hline $\begin{array}{l}\text { Vasodilatadores diretos e } \\
\text { vasoprotetores }\end{array}$ & 0,4 & 2 & 0,6 & 3 & 0,3 & 1 \\
\hline$\beta$-bloqueadores & 3,5 & 17 & 4,3 & 20 & 4,6 & 17 \\
\hline Bloqueadores do canal de cálcio & 3,2 & 16 & 4,9 & 23 & 7,6 & 28 \\
\hline $\begin{array}{l}\text { Fármacos que agem no sistema } \\
\text { renina-angiotensina }\end{array}$ & 10,6 & 52 & 12 & 56 & 17,3 & 64 \\
\hline Antilipêmicos & 3,5 & 17 & 4,1 & 19 & 4 & 15 \\
\hline $\begin{array}{l}\text { G - Sistema genitourinário e } \\
\text { hormônios sexuais }\end{array}$ & 1,8 & 9 & 1,7 & 8 & 1,3 & 5 \\
\hline $\begin{array}{l}\text { Hormônios sexuais, } \\
\text { moduladores do sistema genital } \\
\text { e fármacos urológicos }\end{array}$ & 1,8 & 9 & 1,7 & 8 & 1,3 & 5 \\
\hline $\begin{array}{l}\text { H - Preparações sistema } \\
\text { hormonal excluindo hormônios } \\
\text { sexuais e insulina }\end{array}$ & 2,4 & 12 & 1,7 & 8 & 2,2 & 8 \\
\hline $\begin{array}{l}\text { Corticoesteróides e fármacos } \\
\text { utilizados em terapia tireoidiana }\end{array}$ & 2,4 & 12 & 1,7 & 8 & 2,2 & 8 \\
\hline J - Antiinfecciosos sistêmicos & 1,2 & 6 & 0,2 & 1 & & \\
\hline Antibacterianos de uso sistêmico & 1,2 & 6 & 0,2 & 1 & & \\
\hline $\begin{array}{l}\text { L - Antineoplásicos e agentes } \\
\text { imunomoduladores }\end{array}$ & 0,6 & 3 & 0,2 & 1 & 0,3 & 1 \\
\hline Terapia antineoplásica endócrina & 0,6 & 3 & 0,2 & 1 & 0,3 & 1 \\
\hline M - Sistema músculo esquelético & 8,1 & 40 & 8,8 & 41 & 5,7 & 21 \\
\hline $\begin{array}{l}\text { Antiinflamatórios, } \\
\text { antirreumáticos e antigotosos }\end{array}$ & 4,3 & 21 & 4,1 & 19 & 1,3 & 5 \\
\hline Relaxantes musculares & 1,2 & 6 & 1,5 & 7 & 0,3 & 1 \\
\hline Bisfosfanatos & 2,6 & 13 & 3,2 & 15 & 4,1 & 15 \\
\hline $\mathrm{N}$ - Sistema nervoso & 8,3 & 41 & 9,2 & 43 & 7,3 & 27 \\
\hline Analgésicos & 1,8 & 9 & 1,7 & 8 & 0,3 & 1 \\
\hline Antiepilépticos e psicolépticos & 1,4 & 7 & 1,3 & 6 & 0,8 & 3 \\
\hline Psicoanalépticos & 4,5 & 22 & 5,1 & 24 & 5,4 & 20 \\
\hline Antivertiginosos & 0,6 & 3 & 1,1 & 5 & 0,8 & 3 \\
\hline R - Sistema respiratório & 3,2 & 16 & 1,7 & 8 & 2,2 & 8 \\
\hline Preparações nasais & 0,8 & 4 & 0,6 & 3 & 1,1 & 4 \\
\hline Antiasmáticos & 0,6 & 3 & 0,9 & 4 & 1,1 & 4 \\
\hline $\begin{array}{l}\text { Preparações p/ resfriado, tosse e } \\
\text { antihistamínicos de uso sistêmico }\end{array}$ & 1,8 & 9 & 0,2 & 1 & & \\
\hline S - Órgãos sensoriais & 1 & 5 & 1,7 & 8 & & \\
\hline Preparações oftamológicas & 1 & 5 & 1,7 & 8 & & \\
\hline Outros & 14,2 & 70 & 9,4 & 44 & 6,5 & 24 \\
\hline Total & 100 & 492 & 100 & 467 & 100 & 370 \\
\hline
\end{tabular}


mundial de promoção racional para o uso de medicamentos, ainda sefaz necessário implementar estratégias para melhorar aspectos relacionados à prescrição e à assistência ao paciente. Idosos, em especial do sexo feminino, estão mais propensos à farmacoterapêutica uma vez que a prescrição de medicamentos é a forma de intervenção mais frequente ${ }^{33}$. Na presente pesquisa, a prevalência do uso de medicamentos pelas idosas, nos dois momentos (prée pós-intervenção), foi maior que em alguns estudos nacionais, como os realizados em Bambuí ${ }^{34}$, no município de Santa Rosa-RS ${ }^{35}$, em Belo Horizonte ${ }^{36}$ e na região urbana de Fortaleza ${ }^{37}$, e bem próxima ao encontrado em outros estudos brasileiros ${ }^{6,38}$. M esmo mantendo uma alta taxa de consumo após a intervenção interdisciplinar, em torno dos

Tabela 2 . Prevalência das patologias autoreferidas na amostra de pacientes idosas investigadas.

\begin{tabular}{lcc}
\hline \multirow{2}{*}{\begin{tabular}{l} 
Patologia \\
\cline { 2 - 3 }
\end{tabular}} & $\begin{array}{c}2005 \\
(\mathrm{n}=130)^{*}\end{array}$ & $\begin{array}{c}2006 / 2007 \\
(\mathrm{n}=130)^{*}\end{array}$ \\
\hline Hipertensão arterial & 90 & 113 \\
Osteoporose & 16 & 37 \\
Dislipidemia & 16 & 10 \\
Diabetes melito & 15 & 17 \\
Depressão & 9 & 30 \\
Insuficiência cardíaca & 8 & 8
\end{tabular}

${ }^{*} \mathrm{n}=$ número total deidosas atendidas.
$85 \%$, foi priorizado neste estudo que cada idosa recebesse a melhor indicação terapêutica, sendo ela farmacológica ou não, de acordo com as suas características individuais, tais como as fisiopatológicas, as sócio-econômicas ou as psicológicas. A média demedicamentos por prescrição foi superior aos dados encontrados em estudos ${ }^{4,39,40}$ sobre o consumo de medicamentos quando a população investigada não envolvia somenteidosos, salientando-se que esta geração consome mais medicamentos. Com relação a trabalhos sobre esta população específica, a média de consumo de medicamentos no momento pré-intervenção foi semelhante ao trabal ho de N óbrega et al. ${ }^{41}$ e menor no momento pós-intervenção ${ }^{5,42}$. Torna-se relevante ressaltar que a média de medicamentos por prescrição diminuiu significativamente após a intervenção, o que demonstra uma redução na quantidade individual de consumo. Porém, estes resultados reforçam que idosos consomem muitos medicamentos, configurando que a população de mulheres idosas investigadas permanece polimedicada, uma vez que a Organização Mundial de Saúde preconiza de 1,3 a 2,2 medicamentos por prescrição ${ }^{43}$. A prevalência de polifarmácia, tanto na pré-intervenção como na pós-intervenção, foi superior aos encontrados em estudo nacional ${ }^{44}$ e internacionais ${ }^{30,45}$. Porém, a polimedicação maior, após a intervenção foi inferior ao encontrado em países desenvolvidos ${ }^{45}$ e semelhante ao encontrado no Brasil ${ }^{44}$. Embora a redução na quantidadedeido-

Tabela 3. Indicadores de prescrição da promoção do U RM na amostra de pacientes idosos investigados. Razão média entre consumo médio e as variáveis dos indicadores investigados

\begin{tabular}{|c|c|c|c|}
\hline \multicolumn{4}{|c|}{ Indicadores de prescrição } \\
\hline \multirow[b]{2}{*}{ Indicadores } & \multicolumn{2}{|c|}{ Pré-intervenção } & \multirow{2}{*}{$\frac{\text { Pós-intervenção }}{2006 / 2007}$} \\
\hline & 2005 & $2006 / 2007$ & \\
\hline Consumo médio (medicamentos/prescrição) & $\begin{array}{c}4,4^{\alpha}( \pm 2,9) \\
(n=492)\end{array}$ & $\begin{array}{c}4,2^{\alpha}( \pm 2,6) \\
(n=467)\end{array}$ & $\begin{array}{l}3,1^{\alpha^{*}}( \pm 2,0) \\
(n=370)\end{array}$ \\
\hline$\%$ de medicamentos prescritos por nome Genérico & $\begin{array}{c}10,8(n=53) \\
0,1^{\alpha}\end{array}$ & $\begin{array}{c}24,0(n=112) \\
0,2^{\alpha}\end{array}$ & $\begin{array}{c}42,97(n=159) \\
0,5^{*}\end{array}$ \\
\hline $\begin{array}{l}\% \text { de medicamentos prescritos contidos na WHO } \\
\text { formulary }\end{array}$ & $\begin{array}{c}47,8(n=235) \\
0,4^{\beta}\end{array}$ & $\begin{array}{c}45,4(n=212) \\
0,4^{\beta}\end{array}$ & $\begin{array}{c}57,8(n=214) \\
0,6^{\beta *}\end{array}$ \\
\hline$\%$ de medicamentos prescritos contidos na REN AM E & $\begin{array}{c}58,1(n=286) \\
0,5^{\beta}\end{array}$ & $\begin{array}{c}55,7(n=260) \\
0,5^{\beta}\end{array}$ & $\begin{array}{c}68,6(n=254) \\
0,7^{* *}\end{array}$ \\
\hline$\%$ de medicamentos prescritos contidos nos critérios & $9,6(n=47)$ & $7,5(n=35)$ & $5,1(n=19)$ \\
\hline
\end{tabular}
de Beers 
sas polimedicadas não tenha ocorrido de forma significativa, a interven ção demonstrou ser efetiva quando diminuiu o índice de polimedicação mai or. A redução de eventos de medicação diminui os custos com produtos e serviços de saúdee 0 risco de reações adversas ${ }^{46}$.

Os princípios ativos mais utilizados pelas idosas do estudo estão em concordância com as patologias mais prevalentes nesta parcela da população, com os distúrbios cardiovasculares sendo os mais encontrados. Outras doenças presentes foram a osteoporose, a diabetes melito e a depressão, em concordância também com outros estudos ${ }^{47-49}$. Em estudo semelhante realizado com a população brasileira no Distrito Federal, encontrou-se $54,2 \%$ de síndrome metabólica ${ }^{50}$. 0 quadro clínico geral do grupo investigado demonstra a importância de se ter uma abordagem interdisciplinar na reflexão sobre a conduta clínica.

Em relação às listas padronizadas de medicamentos (WHO formulary e RENAM E), os resultados encontrados, mesmo após a intervenção, apontam uma aceitação abaixo do relatado em outros estudos ${ }^{51,52}$. A padronização de formulários e listas visa elencar os medicamentos essenciais, o que pressupõem que estes produtos vão atender a maioria das necessidades da população, já quese baseiam no perfil epidemiológico de doenças. No entanto, a população idosa, maior consumidora de medicamentos, nem sempre é contemplada de forma satisfatória em tais listas. Os medicamentos propostos como primeira escolha pelas diretrizes de doenças crônicas e seguros para idosos nem sempre estavam presentes na lista nacional de medicamentos essenciais e, portanto, não eram dispensados pelo sistema público de saúde, dificultando a sua acessibilidade. É digno de nota que, durante o processo de interven ção, tivemos que alterar a prescrição dos medicamentos devido à falta dos mesmos nos postos de dispensação (dados não mostrados), conforme comumente relatado na literatura ${ }^{53,54}$. Desta forma, este cenário constitui-se em uma dificuldade para a terapêutica associada a todos os preceitos do U so Racional dos M edicamentos.

Observou-se um aumento nas prescrições de genéricos ao comparar-se os dois momentos pré intervenção com o pós-intervenção, demonstrando que de maneira geral, os prescritores estão modificando os seus hábitos de prescrição. Cabe ressaltar que houve uma tendência de aumento de prescrição de genéricos da primeira para a segunda consulta na pré-intervenção, comportamento que pode ser justificado pelo fato da prescrição médica eodontológica ser obrigatoriamen- te realizada pela denominação comum brasileira, no âmbito do SistemaÚ nico de Saúde (SUS).

Com relação ao consumo de medicamentos impróprios para idosos os valores encontrados foram menores, porém não menos graves, que estudos realizados com a população idosa em ambientes hospitalares e Instituições de Longa Permanência ${ }^{55,56}$. A relação entre as idosas polimedicadas e aquelas que consumiam pelo menos um medicamento impróprio foi significativa assim como em outras pesquisas ${ }^{57}$. A tendência à redução de medicamentos contidos no critério de Beers ${ }^{28,29}$, durante os momentos pré-intervenção, pode ter ocorrido em razão de uma conscientização dos prescritores do SUS com a segurança farmacoterapêutica em idosos. N óbrega \& Karnikowski ${ }^{11}$ sinalizaram alguns medicamentos disponibilizados no mercado brasileiro que se encontravam entre os considerados impróprios aos idosos. Porém, a presença de alguns destes medicamentos permaneceu, mesmo após a revisão da REN AM E realizada em 2006, diminuindo as opções terapêuticas. Cabe ressaltar que em alguns casos existe a necessidade de utilização destes medicamentos impróprios por constituírem-senaúnica opção terapêutica, como ocorre com o idoso que possui I nsuficiência Cardíaca e Fibrilação Atrial, quando o benefício da utilização de digitálicos supera os seus riscos.

0 tempo médio de consulta médica e de retorno encontrados neste estudo esteve acima do proposto pela OMS, refletindo a qualidade da atenção prestada ao paciente durante todos os atendimentos ${ }^{58}$.

Verificou-se nesta pesquisa que a automedicação écorrente en tre as idosas, o que pode contribuir para o surgimento de Problemas Relacionados a M edicamentos, em específico às interações medicamentosas e reações adversas ${ }^{33,59}$. A literatura ${ }^{12,60}$ aponta um índice de automedição maior do que o encontrado no corrente estudo, provavelmente devido à análise nesta pesquisa ter sido realizada somente para medicamentos utilizados de forma contínua.

Enfatizando a efetividade da intervenção interdisciplinar realizada no atual trabalho, Rollason e Vogt ${ }^{61}$, em revisão sistemática, avaliaram estudos que tinham como objetivo a redução do número de medicamentos utilizados, por idosos, através de intervenções com médicos e farmacêuticos. Eles observaram resultados semeIhantes aos encontrados na presente pesquisa, a qual detectou uma redução na média de medicamentos por prescrição, quando comparado 0 período pré-intervenção com o pós-intervenção. 
0 presenteestudo apresenta limitações. Dentre as principais, destaca-se que apesar de todos os profissionais envolvidos realizarem orientações sobre as indicações dos medicamentos prescritos, não foi possível avaliar a efetividade destas orientações. O utro aspecto a ser considerado é que embora tenha sido constatado o hábito da automedicação entre as idosas, também não foi possível avaliar se a intervenção foi efetiva na diminuição desta prática.

Os resultados obtidos permitiram verificar que a interven ção interdisciplinar, envolvendo médicos, farmacêuticos e nutricionistas, pôde contribuir para melhorar os indicadores de U so Racional dos Medicamentos, em especial os de prescrição, para a população idosa. A intervenção interdisciplinar direcionada a esta faixa etá- ria pode contribuir para a promoção do Uso Racional de M edicamentos, seconstituindo num caminho para rever a setorização dos saberes.

\section{Colaboradores}

EFF M edeiros, CF M oraes, M Karnikowski, OT Nóbrega e MGO Karnikowski participaram, igualmente, de todas as etapas de elaboração do artigo.

\section{Referências}

1. Dominguez LJ, Galioto A, Ferlisi A, Pineo A, Putignano $E$, Belvedere $M$, Costanza $G$, Barbagallo $M$. Ageing, lifestyle modifications, and cardiovascular disease in developing countries. J Nutr Health Aging 2006; 10(2): 143-149.

2. Brasil. M inistério do Orçamento e Gestão. Instituto Brasileiro de Geografia e Estatística. Pesquisa Nacional por Amostra de Domicílios (PNAD): Síntese de indicadores de 2008. [acessado 2009 maio 14]. Disponível em: ftp://ftp.ibge.gov.br/Indicadores_Sociais/ Sintese de Indicadores Sociais 2008/Tabelas/

3. Veras R. Em busca de uma assistência adequada à saúde do idoso: revisão da literatura e aplicação de um instrumento de detecção precoce e de previsibilidade de agravos. Cad Saude Publica 2003;

4. 19(3):705-715.

Fleith VD, Figueiredo MA, Figueiredo KFLSRO, Moura EC. Perfil de utilização de medicamentos em usuários da rede básica de saúde de Lorena, SP. Cien Saude Colet 2008; 13(Supl.):755-762.

5. Ribeiro AQ, Rozenfeld S, Klein CH, César CC, Acurcio FA. Inquérito sobre uso de medicamentos por idosos aposentados, Belo Horizonte, M G. Rev Saude Publica 2008; 42(4):724-732.

6. Frazier SC. Health outcomes and polypharmacy in elderly individuals: an integrated literature review. J Gerontol Nurs 2005; 31(9):4-11.

7. Salazar JA, Poon I, Nair M. Clinical consequences of polypharmacy in elderly: expect the unexpected, think the unthinkable. Expert Opin Drug Saf 2007; 6(6):695-704

8. M osegui GBG, Rozenfeld S, Veras RP, Vianna CM M Avaliação da qualidade do uso de medicamentos em idosos. Rev Saude Publica 1999; 33(5):437-444.
9. Fernández-Llimós F, Tuneu L, Baena MI, Garcia Delago A, Faus MJ. Morbidity and Mortality Associated with Pharmacotherapy. Evolution and Current Concept of Drug-Related Problems. Curr Pharm Des 2004; 10:3947-3967.

10. Cipolle RJ, Strand LM, Morley PC. 0 exercício do cuidado farmacêutico. Brasília: Conselho Federal de Farmácia; 2006.

11. Nóbrega OT, Karnikowski M GO. A terapia medicamentosa no idoso: cuidados na medicação. Cien Saude Colet 2005; 10(2):309-313.

12. Bortolon PC, Medeiros EFF, Naves JOS, Karnikowski MGO, Nóbrega OT. Análise do perfil de automedicação em mulheres idosas brasileiras. Cien Saude Colet 2008; 13(4):1219-1226.

13. Medeiros EFF, Bortolon PC, Nóbrega OT, Karnikowski M GO. Problemas relacionados aos medicamentos: morbi-mortalidade em idosos. In. N óbrega OT, Karnikowski MGO, organizadores. Ciência, Saúde e Envelhecimento. Brasília, Editora Universa; 2007.

14. Gomes, CAPG, Fonseca AL, Santos FJP, Rosa MB, M achado MC, Fassy M F. A Assistência Farmacêutica na Atenção À Saúde. Belo Horizonte: FUNED; 2007.

15. World Health Organization (WHO). Promoting rational use of medicines: core components. WHO Policy Perspectives on M edicines, $n^{\circ} 5$. Geneva: WHO; 2002.

16. Leite $S N$, Vieira M, Veber AP. Estudos de utilização de medicamentos: uma síntese de artigos publicados no Brasil e América Latina. Cien Saude Colet 2008; 13(Supl.):793-802. 
17. M elo DO, Ribeiro E, Storpirtis S. A importância e a história dos estudos de utilização de medicamentos. Rev Bras Cienc Farm 2006; 42(4):475-485.

18. Frigotto GA. Interdisciplinaridade como necessidade e como problema nas ciências sociais. In: Bianchetti L, Jantsch A. Interdisciplinaridade: Para além da filosofia do sujeito. 6 a ed. Petrópolis: Editora Vozes; 2002.

19. M otta LB, Aguiar AC. N ovas competências profissionais em saúde e o envelhecimento populacional brasileiro: integralidade, interdisciplinaridade e intersetorialidade. Cien Saude Colet 2007; 12(2):363-372.

20. Brasil. Ministério da Saúde. Agência Nacional de Vigilância Sanitária: Resolução de Diretoria Colegiada n² 268. Brasília: M S; 2003.

21. World Health Organization/WHO Collaborating Centre for Drug Statistics M ethodology. Anatomical therapeutic chemical (ATC) classification index-including defined daily doses (DDDs) for plain substances. Oslo:WHO-Oslo, 2008. [cited 2008 maio 17]. Available from: http://www.whocc.no/atcddd

22. M anagement Sciences for $\mathrm{H}$ ealth (MSH). M anaging Drug Suply. 2 a ed. Connecticut: Kumarian Press; 1997.

23. Bjerrum L, Søgaard J, Hallas J, Kragstrup J. PoIypharmacy: correlations with sex, age and drug regimen. A prescription database study. Eur J Pharmacol 1998; 54(3):197-202.

24. World Health Organization (WHO). How to investigate drug use in health care facilities. Geneva, World Health Organization; 1993.

25. Brasil. Lei 9787 de 10 de fevereiro de 1999. Altera a lei $n^{\circ} 6360$ de 23 de setembro de 1976, que dispõe sobre a vigilância sanitária, estabelece o medicamento genérico, dispõe sobre a utilização de nomes genéricos em produtos farmacêuticos e dá outras providências. Diário O ficial da União; 1999.

26. World Health Organization (WHO). Who Model Formulary. Based in the $15^{\text {th }}$ Model List of Essencial M edicines 2007. Genebra: WHO; 2008.

27. Brasil. M inistério da Saúde. Secretaria de Políticas de Saúde. Departamento de Atenção Básica. Gerência Técnica de Assistência Farmacêutica. Relação $\mathrm{N}$ acional de Medicamentos Essenciais: REN AM E/Secretaria de Políticas de Saúde, Departamento de Atenção Básica, Gerência Técnica de Assistência Farmacêutica. Brasília: Ministério da Saúde; 2006.

28. Beers MH. Explicit criteria for determining potentially inappropriate medication use by the elderly: an update. Arch Intern Med 1997; 157(14):15311536.

29. Fick DM, Cooper JW, Wade WE, Waller J L, Maclean JR, Beers M H. U pdating the Beers Criteria for Potentially Inappropriate Medication U se in Older Adults-Results of a US Consensus Panel of Experts. Arch Intern M ed 2003; 163:2716-2724.

30. Loyola Filho AI, U choa E, Firmo JOA, Lima-Costa $M F$. Influência da renda na associação entre disfunção cognitiva e polifarmácia: Projeto Bambuí. Rev Saude Publica 2008; 42(1):89-99.

31. Alam K, Mishra P, Prabhu M, Shankar PR, Palaian S, Bhandari RB, Bista D. A study on rational drug prescribing and dispensing in outpatients in a tertiary care teaching hospital of Western Nepal. Kathmandu U niv M ed J 2006; 4(16):436-443.
32. Awad AI, Ball DE, Eltayeb IB. Improving rational drug use in Africa: the example of Sudan. Eastn M editerr Health J 2007; 13(5):1202-1211.

33. M clean AJ, Le Couteur DG. Aging biology and geriatric clinical pharmacology. Pharmacol Rev 2004; 56(2):163-184.

34. Loyola Filho AI, Uchoa E, Firmo JOA, Lima-Costa MF. Estudo de base populacional sobre o consumo de medicamentos entre idosos: Projeto Bambuí. Cad Saude Publica 2005; 21(2):545-553.

35. Flores VB, Benvegnú LA. Perfil de utilização de medicamentos em idosos da zona urbana de Santa Rosa, Rio Grande do Sul, Brasil. Cad Saude Publica 2008; 24(6):1439-1446.

36. Loyola Filho Al, U choa E, Lima-Costa M F. Estudo epidemiológico de base populacional sobre uso de medicamentos entre idosos na Região M etropolitana de Belo Horizonte, Minas Gerais, Brasil. Cad Saude Publica 2006; 22(12):2657-2667.

37. Coelho Filho JM, Marcopito LF, Castelo A. Perfil de utilização de medicamentos por idosos em área urbana do Nordeste do Brasil. Rev Saude Publica 2004; 38(4):557-564.

38. Flores LM, M engue SS. Uso de medicamentos por idosos em região do sul do Brasil. Rev Saude Publica 2005; 39(6):924-929.

39. Bertoldi AD, Barros AJD, Hallal PC, Lima RC. Utilização de medicamentos em adultos: prevalência e determinantes individuais. Rev Saude Publica 2004; 38(2):228-238.

40. Keohavong B, Syhakhang L, Sengaloundeth S, Nishimura A, Ito K. Rational use of drugs: prescribing and dispensing practices at public health facilities in Lao PDR. Pharmacoepidemiol Drug Saf 2006; 15(5): 344-347.

41. Nóbrega OT, M elo GF, Karnikowski M GO. Pattern of drugs prescribed for community-residing middle-aged and older adults from the outskirts of Brasília. Rev Bras Cienc Farm 2005; 41(2):271-277.

42. Williams M E, Pulliam CC, Hunter R, Johnson TM, Owens JE Kincaid J, Porter C, Koch G. The ShortTerm Effect of Interdisciplinary Medication Review on Function and Cost in Ambulatory Elderly People. J Am Geriatr Soc 2004; 52(1):93-98.

43. World Health Organization (WHO). Indicators for monitoring National Drug Policies, Action Programme on Essencial Drugs. Geneva; 1994.

44. Veehof LJG, Stewart RE, H aaijer-Ruskamp FM, Jong $B M$. The development of polypharmacy. A longitudinal study. Fam Pract 2000; 17(3):261-267.

45. Hajjar ER, Cafiero AC, Hanlon JT. Polypharmacy in Elderly Patients. Am J Geriatric Pharmacother 2007; 5(4):345-351.

46. Zarowitz BJ, Stebelsky LA, M uma BK, Romain TM, Peterson EL. Reduction of high-risk polypharmacy drug combinations in patients in a managed care setting. Pharmacothery 2005; 25(11):1636-1645.

47. Cabrera MAS, Jacob-Filho W. Obesidade em idosos: prevalência, distribuição e associação co hábitos e co-morbidades. Arq. Bras. Endocrinol Metab. 2001; 45(5):494-501. 
48. Lima-Costa M F, Barreto SM, Giatti L. Condições de saúde, capacidade funcional, uso de serviços e gastos com medicamentos da população idosa brasileira: um estudo descritivo baseado na Pesquisa Nacional por Amostra de Domicílios. Cad Saude Publica 2003; 19(3):735-743.

49. Piccini RX, Fachini LA, Tomasi E, Thumé E, Silveira DS, Siqueira DS, Siqueira FV, Rodrigues MA. Necessidade de saúde comuns aos idosos: efetividade na oferta e utilização em atenção básica à saúde. Cien Saude Colet 2006; 11(3):657-667.

50. Silveira SRP, N óbrega OT, Almeida JLT, Karnikowski M GO, Lottenberg AM P, Silva APR. Parâmetros metabólicos e inflamatórios em uma população de idosas portadoras e não portadoras da Síndrome M etabólica. [tese]. Brasília: UnB; 2007.

51. Cunha MCN, Zorzatto JR, Castro LLC. Avaliação do uso de medicamentos na Rede Pública M unicipal de Saúde de Campo Grande/M S. Rev Bras Cienc Farm 2002; 38(2):217-227.

52. Santos V, Nitrini SM OO. Indicadores do uso de medicamentos prescritos e de assistência ao paciente de serviços de saúde. Rev Saude Publica 2004; 38(6):819-826.

53. Karnikowski M GO, Nóbrega OT, Naves JOS, Silver LD. Access to Essential Drugs in 11 Brazilian Cities: A Community-based Evaluation and Action Method. J Public Health Policy 2004; 25(3-4):288-298.

54. Naves JOS, Silver LD. Evaluation of pharmaceutical assistance in public primary care in Brasília, Brazil. Rev Saude Publica 2005; 39(2):223-230.

55. Barry P J, O'Keefe N, O'Connor KA, O'M ahony D. Inappropriate prescribing in the elderly: a comparison of the Beers criteria and the improved prescribing in the elderly tool (IPET) in acutely ill elderly hospitalized patients. J Clinic Pharm Ther 2006; 31(6):617-626.

56. Castellar JI, Karnikowski M GO, Vianna LG, Nóbrega OT. Estudo da farmacoterapia prescrita a idosos em Instituição Brasileira de Longa Permanência. Acta M ed Port 2007; 20:97-105.

57. Hanlon JT, Artz M B, Pieper CF, Lindblad Cl, Sloane RJ, Ruby CM, Schmader KE. Inappropriate medication use among frail elderly inpatients. Ann Pharmacother 2004; 38(1):9-14.

58. Organização Mundial de Saúde (OMS). Guia do instrutor em práticas da boa prescrição médica. Núcleo de Assistência Farmacêutica (NAF/DCB/ ENSP) FIOCRUZ). Genebra: OM S; 2001.

59. Romano-Lieber NS, Teixeira J JV, Farhat FCLG, Ribeiro E, Crozatti MTL, Oliveira GSA. Revisão dos estudos de intervenção do farmacêutico no uso de medicamentos por pacientes idosos. Cad Saude Publica 2002; 18(6):1499-1507.

60. Arrais PSD, Coelho HLL, Batista M CDS, Carvalho $M L$, Righi RE, Arnau JM. Perfil da automedicação no Brasil. Rev Saude Publica 1997; 31(1):71-77.

61. Rollason V, Vogt $N$. Reduction of polypharmacy in the elderly: a systematic review of the role of the pharmacist. Drugs Aging 2003; 20(11):817-832.

Artigo apresentado em 14/05/2009

Aprovado em 21/12/2009

Versão final apresentada em 05/01/2010 